\title{
Simulating STM transport in alkanes from first principles
}

\author{
C. Toher, ${ }^{*}$ I. Rungger, and S. Sanvito ${ }^{\dagger}$ \\ School of Physics and CRANN, Trinity College, Dublin 2, Ireland \\ (Received 20 January 2009; revised manuscript received 3 April 2009; published 27 May 2009)
}

\begin{abstract}
Simulations of scanning tunneling microscopy (STM) measurements for molecules on surfaces are traditionally based on a perturbative approach, most typically employing the Tersoff-Hamann method. This assumes that the STM tip is far from the sample so that the two do not interact with each other. However, when the tip gets close to the molecule to perform measurements, its electrostatic interplay with the substrate may generate nontrivial potential distribution, charge transfer, and forces, all of which may alter the electronic and physical structure of the molecule. These effects are investigated with the ab initio quantum transport code SMEAGOL, combining the nonequilibrium Green's-function formalism with density functional theory. In particular, we investigate alkanethiol molecules terminated with either $\mathrm{CH}_{3}$ or $\mathrm{CF}_{3}$ end-groups on gold surfaces for which recent experimental data are available. We discuss the effects connected to the interaction between the STM tip and the molecule, as well as the asymmetric charge transfer between the molecule and the electrodes.
\end{abstract}

DOI: 10.1103/PhysRevB.79.205427

PACS number(s): 73.63.-b, 72.10.Bg, 71.15.Mb

\section{INTRODUCTION}

Scanning tunneling microscopy (STM) (Ref. 1) is an invaluable surface-characterization tool with multiple applications in molecular electronics. Typically, an atomically sharp probing tip constructed from heavy metals such as platinum, tungsten, or iridium scans a substrate by means of a tiny tunneling current. The method can be used as a topographic tool to map the positions of atoms, defects, and molecules on surfaces, as a spectroscopic tool to probe their local density of states, and more recently in its spin-polarized version as an ultrasensitive magnetometer. ${ }^{2}$ Furthermore STM can also be used as an atomic fabrication tool for depositing atoms and molecules on a surface so as to form nanoscale devices. ${ }^{3}$ Additionally, it can be used to manufacture breaking junctions, where a molecule is pulled out of a surface, so that electronic transport measurements can be performed. ${ }^{4,5}$ Indeed, the level of geometric control provided by the STM even allows mechanical gating experiments to be carried out so that a range of different transport regimes can be investigated for the same molecule. ${ }^{6,7}$ In general, both in its topographic and spectroscopical modes, an STM measurement consists of a collection of different $I-V$ curves for different tip-to-sample positions.

In the vast majority of cases, calculations of STM currents are based on Bardeen's perturbative approach to tunneling ${ }^{8}$ and consist of evaluating the tunneling matrix elements between the STM tip and the sample. A simplified form due to Tersoff and Hamann (TH) (Ref. 9) which reduces to the calculation of the local density of states is now mainstream and usually implemented in standard electronic structure codes. The TH scheme assumes that the STM tip is sufficiently far from the molecule so as not to affect its electronic structure. As a result of this assumption, there is no self-consistent evaluation of the potential drop nor of the charge redistribution between the tip and the sample. These methods thus become unreliable when the tip is close to the molecule and deviations from the TH current are expected. It is therefore important to explore, for STM simulations, the use of selfconsistent transport methods ${ }^{10-12}$ such as the nonequilibrium
Green's-function formalism (NEGF) (Ref. 13) combined with $a b$ initio electronic structures obtained from density functional theory (DFT). ${ }^{14,15}$ This is the goal of our work, which is based on the use of the electronic transport code SMEAGOL. ${ }^{10,11}$

It is important to realize that NEGF-DFT is completely complementary to the standard TH scheme. Indeed, it presents problems in the limit of large tip-to-sample separation, where TH is most effective. The main reason for this is rooted in the fact that SMEAGOL employs the numerical implementation of DFT contained in the SIESTA code. ${ }^{16}$ The SIESTA basis set is constructed from numerical orbitals whose radial component is truncated beyond a certain cutoff radius, ${ }^{17}$ and the description of extended vacuum regions becomes a delicate numerical problem. In fact in the extreme case of a vacuum region extending well beyond all the cutoff radii, all matrix elements between the tip and the sample vanish and the tunneling current is identically zero. Improvements can be obtained by populating the vacuum region with empty orbitals (basis functions not associated to a pseudopotential). However for sensitive calculations of tiny tunneling currents the actual position of the empty orbitals can deeply affect the results, most typically by creating spurious current oscillations as a function of the tip-to-sample distance. Therefore we limit our method to tip-to-sample separation smaller than $6 \AA$ for which reliable calculations do not need empty orbitals in the vacuum. We call this limit the quasicontact limit.

We can summarize this discussion by saying that, while the TH approach is ideal for large tip-to-sample separations, the NEGF scheme becomes competitive for problems where the interaction of the sample with the tip is relevant. However when choosing the method for performing STM simulations one has also to remember that the NEGF scheme is considerably more computationally intensive than the $\mathrm{TH}$ one. In fact, to calculate both topographic and $d I / d V$ maps using NEGF it is necessary to repeat a full self-consistent $I-V$ calculation at each spatial mesh point. In contrast the TH approach necessitates only either a single electronic structure calculation or two electronic structure calculations plus a convolution integral if the structure of the tip is considered. ${ }^{18}$ 
An example of experiments where the tip-to-molecule interactions are believed to be important is that performed by Pflaum et al. ${ }^{19}$ in which a monolayer of alkanethiol molecules is deposited on a gold surface, and the transport properties are then probed by using a STM setup. The obtained zero-bias conductance is low, being on the order of $10^{-7} G_{0}$ at zero bias (the quantum conductance $G_{0}$ is defined as $2 e^{2} / h$, with $e$ as the electron charge and $h$ as the Planck constant). The $I-V$ curves are asymmetric, and this asymmetry increases noticeably when the terminating $\mathrm{CH}_{3}$ group is replaced by a $\mathrm{CF}_{3}$ one. It is speculated that the additional asymmetry of the $\mathrm{CF}_{3}$-terminated molecule is due to a rearrangement of the charge distribution near the end of the molecule caused by the high electronegativity of the $\mathrm{F}$ atoms. This in turn generates an electrostatic force between the STM tip and the molecule, the direction of which depends on the electrostatic potential at the tip. Such interaction causes the molecule to be either repelled or attracted by the tip depending on the bias polarity and creates the asymmetry in the $I-V$.

Calculations using the TH method have been performed for pentanethiol molecules on gold. ${ }^{20}$ However, the nature of the TH method requires a large tip-to-molecule separation, and the current obtained in these calculations is an order of magnitude smaller than that observed in the experiments. In this work, the mechanism behind the asymmetry in the $I-V$ curves of $\mathrm{CH}_{3}$ - and $\mathrm{CF}_{3}$-terminated alkanethiol molecules on gold is explored with NEGF-DFT. The issue related to the electrostatic interaction between the tip and the sample is investigated in an approximate way. First we calculate from equilibrium DFT the electrical dipole on the molecules. Then we use classical electrostatic theory to determine the equilibrium position of the molecule with respect to the tip. Finally new transport calculations are carried out for the newly estimated atomic positions.

\section{COMPUTATIONAL METHOD AND NUMERICAL DETAILS}

STM transport measurements are equivalent to calculating the two-probe $I$ - $V$ curve of a molecule sandwiched in between two metallic electrodes. One electrode represents the surface to which the molecule is attached and the other is the STM tip. The NEGF scheme partitions such system into three regions, respectively, the two current/voltage electrodes (leads) and a middle region called the scattering region (SR) (see Fig. 1).

The SR includes both the molecule and a portion of the leads, and it is extended enough so that charge density calculated at the most external atomic layer resembles that of the bulk electrodes. The leads, which are assumed to be periodic crystals in the transport direction, are kept at different chemical potentials $\mu_{1 / 2}=E_{\mathrm{F}} \pm e V / 2$ where $V$ is the applied potential bias. We note that this is somewhat different from STM experiments, where typically the chemical potential of the surface remains fixed while a potential bias is applied to the probe. However, such a difference is simply a redefinition of the absolute position of the energy scale and does not effect the shape of the $I-V$ curve. The SR is described by a

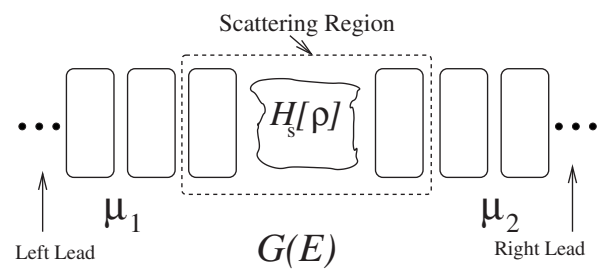

FIG. 1. Schematic diagram of a metal-molecule-metal junction. A scattering region (the part of the system enclosed by the dashed box) is sandwiched between two current/voltage probes kept at the chemical potentials $\mu_{1}$ and $\mu_{2}$, respectively. The electrodes are modeled as being periodic in the direction of transport. A number of layers of the electrodes are included in the scattering region to allow the charge density to converge to the bulk value.

Hamiltonian $H_{s}$. This is used to construct the nonequilibrium Green's function

$$
G(E)=\lim _{\eta \rightarrow 0}\left[(E+i \eta)-H_{s}-\Sigma_{1}-\Sigma_{2}\right]^{-1},
$$

where $\Sigma_{1 / 2}$ are the self-energies for the leads, constructed by semianalytical derivation ${ }^{21}$ from the electronic structure of the bulk. $G(E)$ enters in a self-consistent procedure to calculate the density-matrix $\rho$ of the SR and, hence, the two-probe current of the device. ${ }^{10-13,22}$ The Hamiltonian for the scattering region $H_{\mathrm{s}}$ is generally assumed to be a function of the nonequilibrium charge-density $\rho$, which is calculated following the NEGF prescription as

$$
\rho=\frac{1}{2 \pi} \int_{-\infty}^{\infty} G(E)\left[\Gamma_{1} f\left(E, \mu_{1}\right)+\Gamma_{2} f\left(E, \mu_{2}\right)\right] G^{\dagger}(E) d E,
$$

where $\Gamma_{1 / 2}=i\left[\Sigma_{1 / 2}-\Sigma_{1 / 2}^{\dagger}\right]$. In practice, this integral is performed by splitting it into two parts: ${ }^{10-13,22}$ an equilibrium part to be performed along a contour in the complex plane, and a nonequilibrium part to be evaluated along the real energy axis but that contributes only around $E_{\mathrm{F}}$. Finally, the converged Green's function can be used to calculate the twoprobe current, $I$, through the device

$$
I=\frac{2 e}{h} \int_{-\infty}^{\infty} \operatorname{Tr}\left[G(E) \Gamma_{1} G^{\dagger}(E) \Gamma_{2}\right]\left[f\left(E, \mu_{1}\right)-f\left(E, \mu_{2}\right)\right] d E .
$$

This is effectively the integral between $\mu_{1}$ and $\mu_{2}$ (the bias window) of the transmission coefficients $T(E)$ $=\operatorname{Tr}\left[G(E) \Gamma_{1} G^{\dagger}(E) \Gamma_{2}\right]$.

The NEGF scheme is general and not related to a particular electronic structure theory. In the case of SMEAGOL, ${ }^{10,11}$ the electronic structure method used is DFT (Ref. 14) in the SIESTA implementation. In particular in this work we will use the local density approximation ${ }^{15}$ (LDA) of the exchange and correlation functional.

We use standard scalar-relativistic pseudopotentials with the following reference configurations: $\mathrm{H} 1 s^{1}, \mathrm{C} 2 s^{2} 2 p^{2}$, $\mathrm{S} 3 s^{2} 3 p^{4}$, and $\mathrm{F} 2 s^{2} 2 p^{5}$. A simplified $6 s$-only bandstructure for $\mathrm{Au}$ is used for all the transport calculations in order to reduce the computational overheads. In this case the $\mathrm{Au}$ pseudopotential is constructed with the reference configura- 

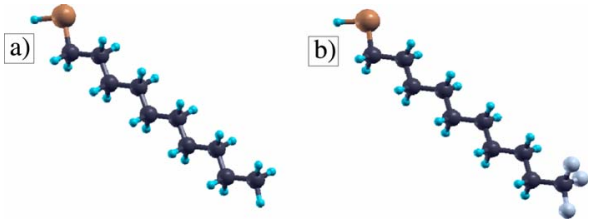

FIG. 2. (Color online) Decanethiol molecule with (a) $\mathrm{CH}_{3}$ and (b) $\mathrm{CF}_{3}$ end-group. Color code: $\mathrm{C}=$ black, $\mathrm{S}=$ dark gray (brown online), $\mathrm{H}=$ light gray (blue online), and $\mathrm{F}=$ dark gray (purple online).

tion $6 s^{1}$, while the $d$ electrons are left in the core and nonlinear core corrections are applied. We note that in general the description of $\mathrm{Au}$ by using $6 s$ orbitals only should be taken with care. However this becomes a necessity here, where the additional computational overheads required by including the $d$ shell in the valence make the calculations impractical. In a previous publication we have investigated the consequences of this drastic approximation on the transport through aromatic rings. We concluded that the effects of having $d$ electrons included explicitly in the Au valence are negligible at least at moderate biases. ${ }^{23}$

The atomic basis set is constructed as follows $\mathrm{H}$ : DZ-s; C: DZP-s, DZP- $p$; S: DZP- $s$, DZP- $p$; F: DZP- $s$, DZP- $p$; and Au: $\mathrm{DZ}-s$ (the notation is $\mathrm{SZ}=$ single zeta, $\mathrm{DZ}=$ double zeta, and $\mathrm{P}=$ polarized). ${ }^{16}$ The real-space grid has an equivalent cutoff energy of $200 \mathrm{Ry}$. The complex part of the integral leading to the charge density is computed by using 60 energy points on the complex semicircle, 48 points along the line parallel to the real axis, and 10 poles. The integral over real energies necessary at finite bias is evaluated over at least 512 points. ${ }^{10,11}$ All the calculations are carried out with periodic boundary conditions in the direction perpendicular to that of the transport and mesh of four $k$ points.

\section{RESULTS}

\section{A. Electronic structure of the molecules}

The molecules used in the experiments of Pflaum et al. ${ }^{19}$ are both methyl-terminated and fluorine-terminated alkanethiols. A range of different alkane chain lengths was used in that study, but most of the data were collected for decanethiols [see Figs. 2(a) and 2(b)]. Decanethiol consists of an alkane chain of ten carbon atoms terminated with a thiol (-SH) group. The sulfur atom in the thiol group forms a strong bond with gold and anchors the molecule to the surface forming a well-ordered self-assembled monolayer.

The orbital resolved density of states (DOS) for isolated decanethiol, both $\mathrm{CH}_{3}$ - and $\mathrm{CF}_{3}$-terminated, is shown in Fig. 3 . The energy gap between the highest occupied molecular orbital (HOMO) and the lowest unoccupied molecular orbital (LUMO) is in both cases rather large $(\sim 5 \mathrm{eV}) \cdot{ }^{24}$ Most importantly the DOSs of the two frontier molecular orbitals do not have large amplitude over the $\mathrm{C}$ atoms forming the decanethiol but are rather localized on the $\pi$ states of $\mathrm{S}$ perpendicular to the molecule axis [see Figs. 3(b) and 3(d)]. In the case of $\mathrm{CF}_{3}$ termination the $\mathrm{F}$ contribution to the DOS is only confined to part of the LUMO and to low-energy HOMO levels. The orbital nature of the HOMO and LUMO

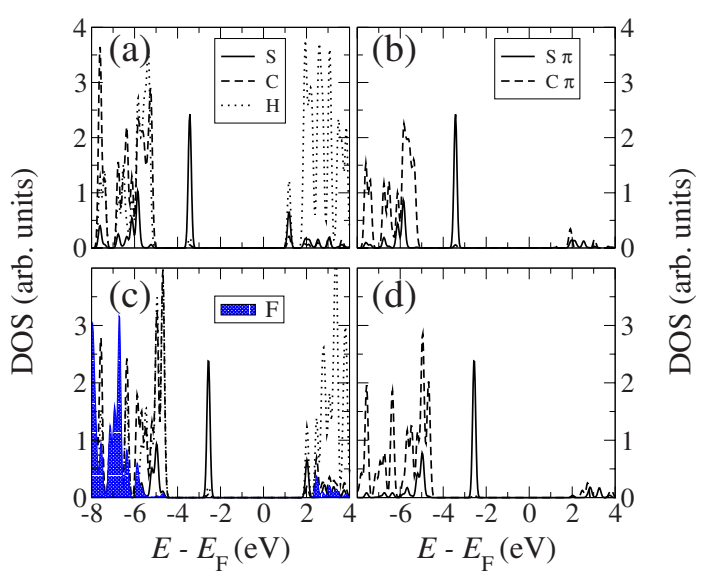

FIG. 3. (Color online) Orbital-resolved DOS for the isolated decanethiol molecule. Panels (a) and (b) are for the $\mathrm{CH}_{3}$ termination, while panels (c) and (d) are for the $\mathrm{CF}_{3}$. In panels (b) and (d) only the DOS corresponding to $\pi$ orbitals perpendicular to the molecule axis are displayed.

is further investigated in Fig. 4 where we present the local DOS for the $\mathrm{CH}_{3}$-terminated molecule (the one for the $\mathrm{CF}_{3}$-terminated case is rather similar and it is not presented here).

We note that both the HOMO and the LUMO have amplitude mainly around the thiol group with little charge spread over the alkane chain. One should therefore expect little conductance through those states. In contrast the first state below the HOMO (HOMO-1) [Fig. 4(a)] appears as a delocalized $\pi$ state and it is expected to conduct efficiently. From this analysis we conclude that the HOMO-1 of the actual molecules corresponds to the delocalized HOMO of the parental polyethylene infinite chain, while the HOMO is associated to the anchoring thiol group.

In order to better understand the transport across decanethiol, in Fig. 5 we present both the real and the complex bandstructures for the parental infinite polyethylene chain, calculated as described in Ref. 21. This has been obtained by inserting $\mathrm{C}_{2} \mathrm{H}_{4}$ into a large supercell, whose lateral dimensions $(10.58 \times 10.58 \AA)$ are enough to isolate the periodic images of the molecule. We have then performed two types of calculations. In the first [Fig. 5(a)] we have included, in addition to the already mentioned basis set, a number of supplementary basis functions in the vacuum region of the
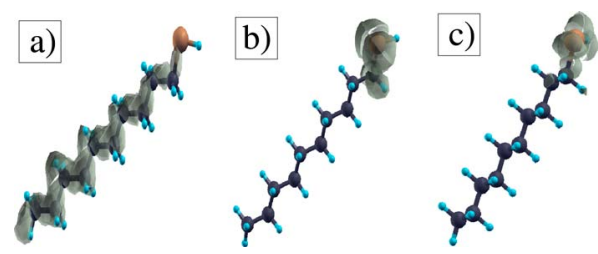

FIG. 4. (Color online) Local density of states for the isolated decanethiol molecule terminated with the $\mathrm{CH}_{3}$ group showing: (a) the HOMO-1 state, (b) the HOMO, and (c) the LUMO. The local DOS for the molecule terminated with the $\mathrm{CF}_{3}$ group is similar and it is not displayed here. Color code: $\mathrm{C}=$ black, $\mathrm{S}=$ dark gray (brown online), $\mathrm{H}=$ light gray (blue online), and $\mathrm{F}=$ dark gray (purple online). 

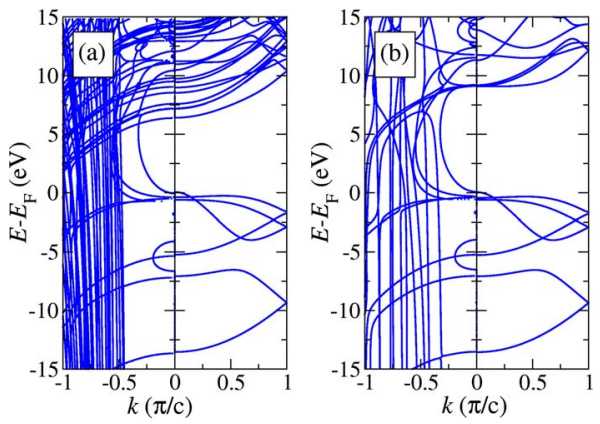

FIG. 5. (Color online) Real (positive $k$ ) and complex (negative $k$ ) bandstructures for a $\mathrm{C}_{2} \mathrm{H}_{4}$ polymer infinite chain: (a) calculations obtained by including basis functions into the vacuum region; (b) calculations obtained with basis functions positioned only over the molecule. Note in both cases the semicircular complex band joining the molecular HOMO and LUMO. Moreover, when basis functions are included into the vacuum paraboliclike bands associated to freeelectron-like states appear in the band gap. The $k$ vector is measured in units of $\pi / c$, where $c=2.5 \AA$ is the supercell lattice vector in the $z$ direction.

supercell. Such basis functions are constructed as DZP-s orbitals by using the $\mathrm{C}$ pseudopotential and they are distributed over a $5 \times 5 \times 3$ mesh in the supercell. The HOMO-LUMO gap for such a calculation is about $6.3 \mathrm{eV}$, a value which agrees reasonably well with that calculated with plane-wave basis set, ${ }^{25}$ but it is considerably smaller than the value of 10 $\mathrm{eV}$ obtained previously with a localized basis set. ${ }^{26}$

The difference is attributed to the fact that the molecular LUMO is actually unbound so that free-electron-like vacuum states appear at midgap. ${ }^{25}$ As a result the actual HOMOLUMO gap of polyethylene chains is between the molecular HOMO and the first vacuum level. Importantly, unless additional basis functions are introduced into the vacuum, algorithms based on localized basis set do not capture such vacuum states. This is shown in Fig. 5(b), where the same calculation is repeated without basis functions in the vacuum. Now the HOMO-LUMO gap is that between levels with amplitude localized entirely over or near the molecule, and it is considerably larger than the HOMO-vacuum gap. This means that such a reduced basis set cannot properly describe vacuum states. The calculations shown in Fig. 5(a) instead use a basis capable of describing energy levels well extended into the vacuum and therefore agree with the planewave results, at least for $\operatorname{small} \operatorname{Im}(k)$ (these are the ones relevant for transport).

Importantly the vacuum levels become irrelevant for the two-probe transport calculations in the bias range described here. In fact the position of the vacuum is set by the Fermi level of the electrodes and their work function. Here the polyethylene HOMO positions are at about $4 \mathrm{eV}$ below $E_{\mathrm{F}}$ (see the next section), which means that the first vacuum level will appear at energies larger than $E_{\mathrm{F}}+\phi_{\mathrm{Au}}$, where $\phi_{\mathrm{Au}}$ is the gold work function. Since $\phi_{\mathrm{Au}}$ is about $5 \mathrm{eV}$ no vacuum states can appear in the bias window for voltages smaller than $5 \mathrm{~V}$. Since here we limit our analysis to voltages smaller than $2 \mathrm{~V}$ (for both the current polarities) we can conclude that vacuum states can be safely neglected. For this reason we do not include any basis functions in the vacuum
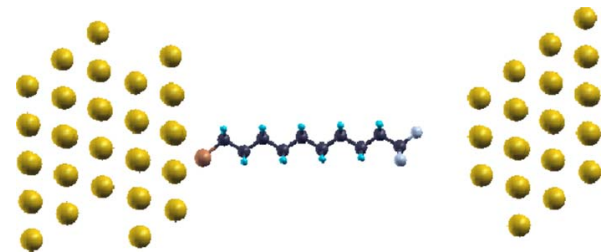

FIG. 6. (Color online) Simulation cell used in the transport calculations: a $\mathrm{CF}_{3}$-terminated decanethiol molecule is attached to gold surface and probed with a Au STM tip. The case of the $\mathrm{CH}_{3}$-terminated molecule is similar and it is not displayed here. Color code: $\mathrm{Au}=$ gray (yellow online), $\mathrm{C}=$ black, $\mathrm{S}=$ dark gray (brown online), $\mathrm{H}=$ light gray (blue online), and $\mathrm{F}=$ dark gray (purple online).

region of the supercell. This last approximation is further validated by the fact that the complex band structure around the Au Fermi level (of HOMO-LUMO nature) is essentially equally well described by a calculation which neglects any basis function in the vacuum [compare Figs. 5(a) and 5(b)].

\section{B. Transport properties of the molecules on gold surfaces}

In order to calculate the transport properties, we attach the molecules to the Au fcc (111) hollow site via a terminating thiol group at a sulfur-surface distance of $1.9 \AA{ }^{27}$ This is the equilibrium distance measured for the hollow site configuration. ${ }^{28}$ The arrangement of the molecule on the surface and the relative position of the tip are shown in Fig. 6 for the $\mathrm{CF}_{3}$-terminated molecule. The configuration for the $\mathrm{CH}_{3}$-terminated case is similar and it is not displayed here. In the experiments from Pflaum et al. ${ }^{19} \mathrm{CH}_{3}$-terminated decanethiols are tilted at an angle of $\sim 32^{\circ}$ from the vertical direction, while for $\mathrm{CF}_{3}$-terminated the tilting angle is $\sim 39^{\circ} .{ }^{19}$ Our simulation cells however have the molecules placed perpendicular to the surface. This allows the unit cell used to be smaller than that required for simulating such tilting angles, and the computational overheads are greatly reduced. We also make some approximations in modeling the STM tip. First of all we consider a gold tip instead of the used tungsten or platinum-iridium ones in such a way to avoid issues related to having two electrodes with different work functions. In doing this we describe the electronic structure of the electrodes with a $6 s$-only basis set, which was proven to provide a reasonably accurate choice for transport calculations at only a moderate computational cost. ${ }^{23,32}$ Second we do not consider an atomically sharp tip but a slightly blunter one. This geometry improves the stability of the calculations, allowing the self-consistent convergence to be quicker and more reliable.

As expected the tunneling current is extremely sensitive to the distance between the tip and the molecule so that it is rather difficult to determine from experiments the precise tip vertical position. Our working strategy is then to adjust this distance to match the magnitude of the current obtained in experiments. Such a tuning exercise is presented in Fig. 7 where we show the $I-V$ curves for different tip-to-molecule separations, where these are defined as the distance between the terminating surface of the tip and the $\mathrm{C}$ atom in the $\mathrm{CH}_{3}$ 

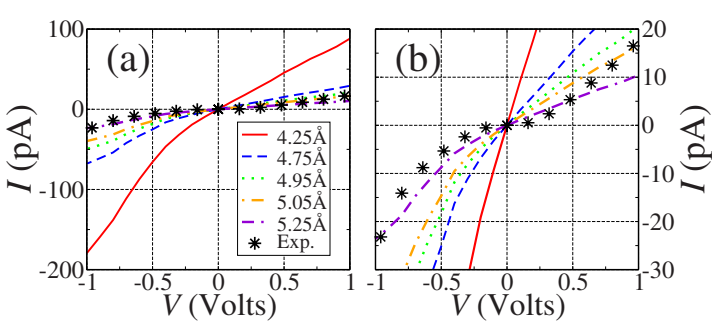

FIG. 7. (Color online) $I-V$ curves for $\mathrm{CH}_{3}$-terminated decanethiol attached to a gold surface for different distances between the $\mathrm{C}$ atom in the $\mathrm{CH}_{3}$ group and the probing tip. Changing this distance by $0.1 \AA$ causes the size of the current to change by approximately one order of magnitude at $1 \mathrm{~V}$. Panel (b) is a zoom of panel (a) in the current range between $[-30,20] \mathrm{pA}$.

group. From the figure one can deduce that the best match is obtained for a distance of $5.25 \AA$. This corresponds to a $\mathrm{Au}-\mathrm{H}(\mathrm{Au}-\mathrm{F})$ distance of $4.62 \AA(4.42 \AA)$, which is about $2 \AA$ longer than the Au-S distance $(2.53 \AA)$ at the thiol termination. The results presented in the rest of this section are obtained for this tip-to-molecule separation.

The orbital resolved DOS for both $\mathrm{CH}_{3^{-}}$and $\mathrm{CF}_{3}$-terminated decanethiol on $\mathrm{Au}$ is shown in Fig. 8. In general and for both the molecules the HOMO, now broadened by the interaction with the surface, is relatively close to the gold $E_{\mathrm{F}}$. However, a closer look at its local DOS (this is calculated as the local DOS over an energy window corresponding to the HOMO level) depicted in Figs. 9 and 10 reveals that such a state is mainly localized around the $\mathrm{S}$ atoms of the thiol groups. The delocalized $\pi$ orbitals capable of efficient charge transport across the molecule (HOMO-1 of the thiol-linked molecules and HOMO of the polyethylene chain) are further away from the Fermi level (approximately $4 \mathrm{eV}$ ). This essentially sets the position of the polyethylene HOMO at about $4 \mathrm{eV}$ below the Au Fermi level, in substantial agreement with previous plane-wave calculations. ${ }^{29}$

It is also interesting to observe the effects that the different terminating groups $\left(\mathrm{CH}_{3}\right.$ and $\left.\mathrm{CF}_{3}\right)$ have over the elec-

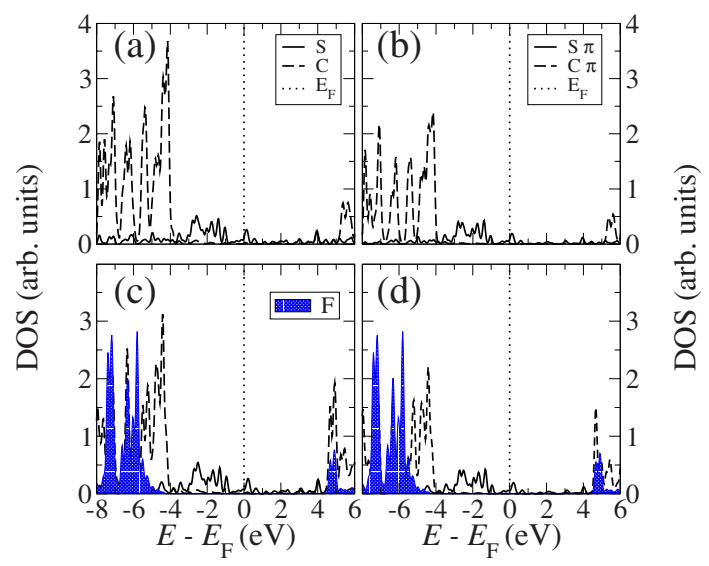

FIG. 8. (Color online) Orbital-resolved DOS for the decanethiol molecule attached to the gold surface. Panels (a) and (b) are for the $\mathrm{CH}_{3}$ termination, while panels (c) and (d) are for the $\mathrm{CF}_{3}$. In panels (b) and (d) only the DOS corresponding to $\pi$ orbitals perpendicular to the molecule axis are displayed for $\mathrm{C}$ and $\mathrm{S}$, while in panel (d) all the fluorine $\pi$ orbitals are shown. The HOMO-LUMO gap is large, although the HOMO is about $1 \mathrm{eV}$ below $E_{\mathrm{F}}$.
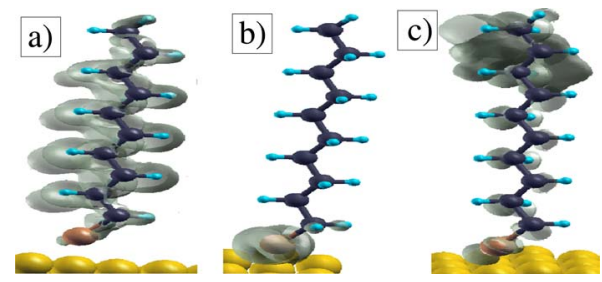

FIG. 9. (Color online) Local DOS isosurface for the $\mathrm{CH}_{3}$-terminated decanethiol molecule on the $\mathrm{Au}$ (111) surface: (a) the HOMO-1 level, (b) the HOMO level, and (c) the LUMO level. Note how the HOMO is localized around the thiol end-group and it is not expected to conduct efficiently. Color code: $\mathrm{Au}=$ gray (yellow online), $\mathrm{C}=$ black, $\mathrm{S}=$ dark gray (brown online), and $\mathrm{H}=$ light gray (blue online).

tronic structure of the HOMO-1 level. The most notable difference is associated with the charge-density distribution along the $\mathrm{CH}_{2}$ groups forming the decanethiol. While in the case of $\mathrm{CH}_{3}$ termination the electron charge distributes almost uniformly over $\mathrm{CH}_{2}$ up to near the terminating $\mathrm{CH}_{3}$ group, in the case of $\mathrm{CF}_{3}$-terminated molecule there is a strong modulation with a decay of the charge density as the $\mathrm{CF}_{3}$ group is approached. This is due to the Coulomb repulsion from the more electronegative $\mathrm{CF}_{3}$ group. Such an electrostatic-driven charge decay is reminiscent of the same effect investigated by STM for molecules on surfaces in the presence of point charges. ${ }^{30}$

Despite these differences the absence of any extended molecular orbital near the Fermi level of gold is common to both molecules, and therefore we expect just a tiny tunnel current at low bias. This can be appreciated by looking at both the transmission coefficients, $T(E)$ (Fig. 11) and the $I-V$ curves (Fig. 12). There is a large gap in the resonances in the zero-bias transmission coefficients of about $5 \mathrm{eV}$ on each side of the Fermi level with the edges of such a gap corresponding to the HOMO-1 (HOMO of polyethylene) and the LUMO, respectively, below and above the Au Fermi energy. The transmission of the HOMO provides only a small shoulder in $T(E)\left(T \sim 10^{-6}\right)$ at about $2.5 \mathrm{eV}$ below $E_{\mathrm{F}}$, confirming that the HOMO does not provide an efficient transport channel at resonance. However, since the HOMO is the first molecular level to enter the bias window (at least for one of the
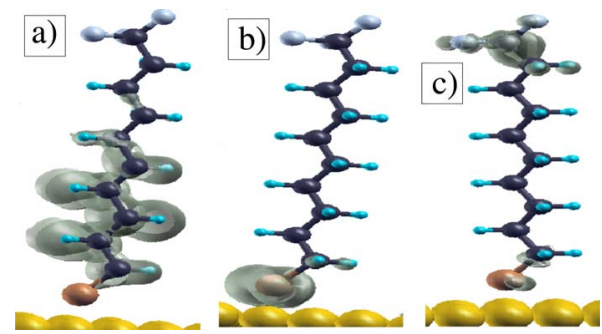

FIG. 10. (Color online) Local DOS isosurface for the $\mathrm{CF}_{3}$-terminated decanethiol molecule on the $\mathrm{Au}$ (111) surface: (a) the HOMO-1 level, (b) the HOMO level, and (c) the LUMO level. Note how the HOMO is localized around the thiol end-group and it is not expected to conduct efficiently. Color code: $\mathrm{Au}=$ gray (yellow online), $\mathrm{C}=$ black, $\mathrm{S}=$ dark gray (brown online), $\mathrm{H}=$ light gray (blue online), and $\mathrm{F}=$ dark gray (purple online). 


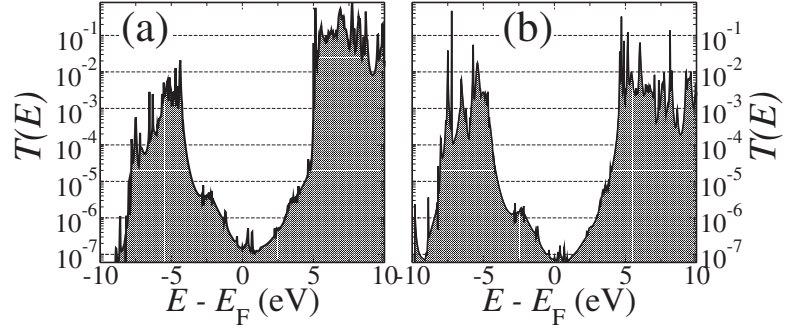

FIG. 11. Transmission coefficients at zero bias for decanethiol attached to gold surface and terminated with (a) $\mathrm{CH}_{3}$ and (b) $\mathrm{CF}_{3}$ end-group. Note the gap in the transmission of about $5 \mathrm{eV}$ on either side of the Fermi level.

two current polarities), it is expected to dominate the lowbias region of the $I-V$.

Moving our attention to the $I-V$ curves and the differential conductances shown in Fig. 12, the most noticeable feature is their asymmetry. In particular conductance at positive bias is between about two to three times smaller than that for negative bias. Moreover we observe that the conductance of $\mathrm{CH}_{3}$-terminated molecules is both larger and more asymmetric than that of $\mathrm{CF}_{3}$-terminated.

The reason for the conductance asymmetry is rooted in the different electronic coupling strength between the molecule and the gold surface as compared to the much weaker one between the molecule and the STM tip. The S atom in fact forms quite a strong bond with the gold surface, whereas the tip is separated by a vacuum region which provides a substantial potential barrier. Such difference in coupling persists to rather close tip-to-molecule separations since both the $\mathrm{CH}_{3}$ and $\mathrm{CF}_{3}$ end-groups do not bind to the Au tip. The mechanism leading to the asymmetry is then illustrated in the cartoon of Fig. 13(a). Because of the different coupling strengths of the two electrodes with the molecule, electrons are easily transferred from the substrate to the molecule, but they find it much more difficult to hop to the tip. This means that the occupation of the molecule is determined by the chemical potential of the substrate and that the molecule gains electronic charges when the electron flux is from the surface to the tip (negative bias according to our definition). In contrast the molecules lose charge when the flux is in the other direction (positive bias). Since charging produces a shift in the molecular electronic states toward higher energies
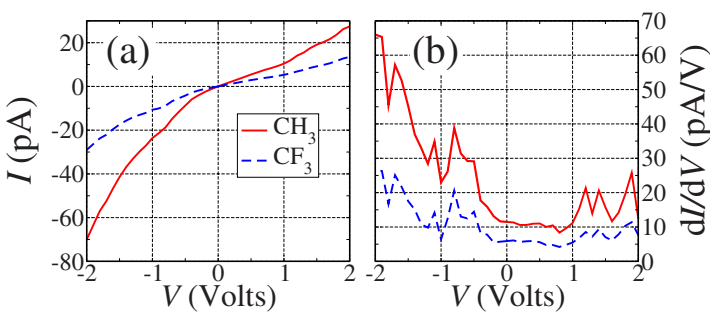

FIG. 12. (Color online) (a) $I-V$ curve and (b) differential conductance for decanethiol molecules terminated with both $\mathrm{CH}_{3}$ and $\mathrm{CF}_{3}$ groups. Note the bias asymmetry, with the conductance at negative bias being two to three times larger than that for positive bias. The conductance is lower for the $\mathrm{CF}_{3}$-terminated molecule than that of the $\mathrm{CH}_{3}$-terminated.

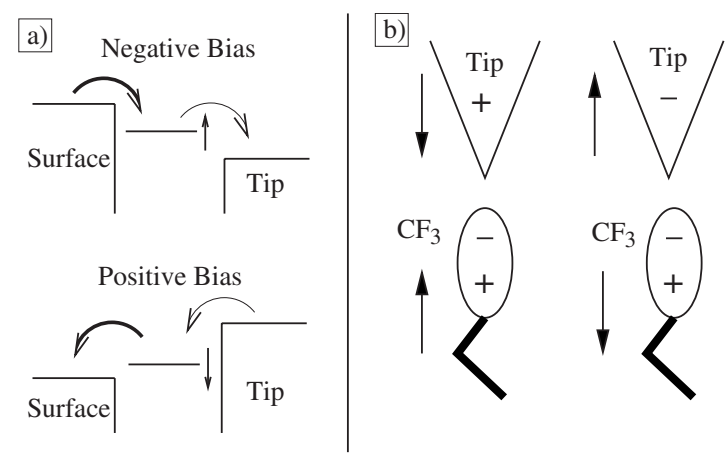

FIG. 13. (a) Schematic energy-level diagram of the STM geometry. Since the molecule is more strongly coupled to the surface than to the tip its occupation is determined by the surface chemical potential; i.e., it will charge for negative bias and decharge for positive. Such a charging mechanism produces a shift of the occupied molecular level toward higher (lower) energy for negative (positive) bias. (b) Cartoon showing the electrostatic interaction between tip and molecule $\mathrm{CF}_{3}$ end-group. In this case there is an electric-dipole forming, so that the molecule will be attracted or repulsed by the STM tip, depending on the bias polarity.

one expects the HOMO to move into the bias window at negative voltages and away from it at positive ones. The same behavior is expected for the HOMO-1, while the LUMO follows exactly the opposite pattern (it moves into the bias window for positive bias and away from it for negative)..$^{31}$

The electrostatic evolution of the system under bias is illustrated in Figs. 14 and 15 where we show the transmission coefficients as a function of energy for different voltages. Clearly the behavior is the one expected by our simple electrostatic model with an upshift (downshift) in energy of the molecular levels at negative (positive) bias. Since the highly conducting HOMO-1 and LUMO levels never enter the bias window in this voltage range (limited to \pm 2 Volt), the onset of the current is solely determined by the position

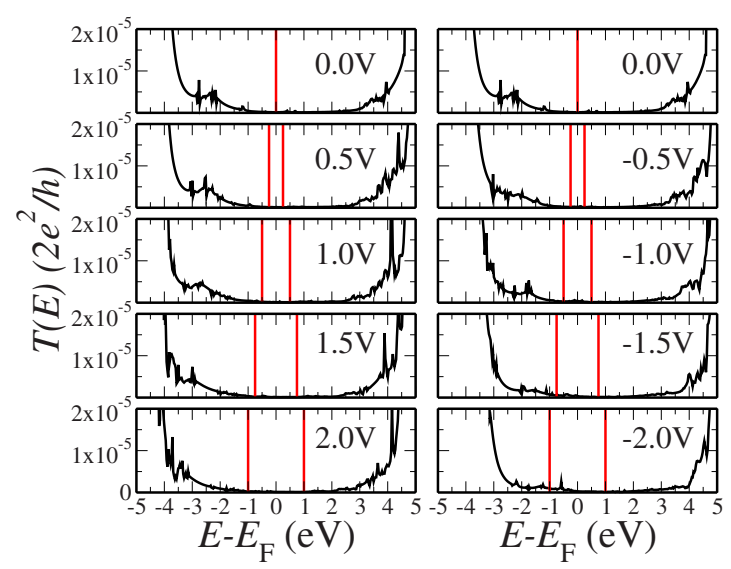

FIG. 14. (Color online) Transmission coefficients as a function of energy for different biases for $\mathrm{CH}_{3}$-terminated decanethiol. Note how the resonances in the transmission coefficients due to the occupied states move up in energy closer to the bias window at negative bias, and move away from the bias window for positive bias. The vertical lines mark the bias window. 


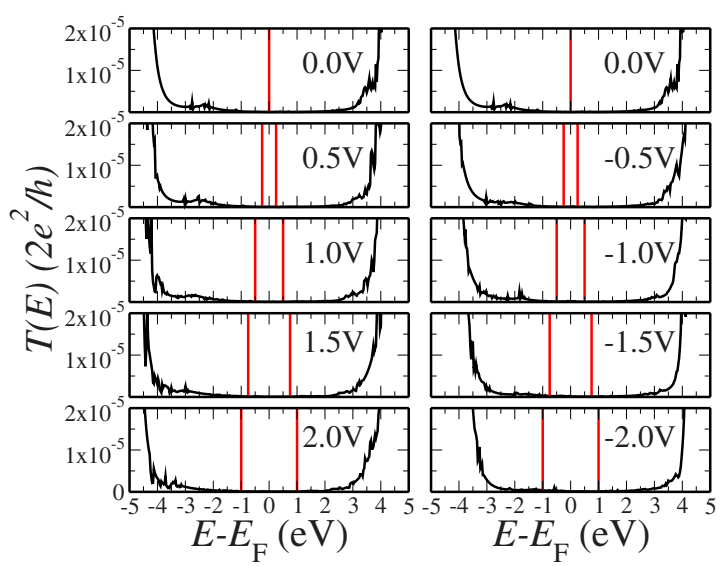

FIG. 15. (Color online) Transmission coefficients as a function of energy for different biases for $\mathrm{CF}_{3}$-terminated decanethiol. Note how the resonances in the transmission coefficients due to the occupied states move up in energy closer to the bias window at negative bias and move away from the bias window for positive bias. The vertical lines mark the bias window.

of the HOMO. This however can enter the bias window only for negative bias explaining the current asymmetry. Furthermore, since the transmission of the HOMO for $\mathrm{CH}_{3}$-terminated decanethiol is larger than that of the $\mathrm{CF}_{3}$-terminated case, the conductance of $\mathrm{CH}_{3}$ decanethiol is both larger and more asymmetric than that of its $\mathrm{CF}_{3}$-terminated counterpart.

Finally, in Fig. 16 we present the total Mülliken population, $N_{M}$, of the molecule as a function of voltage. The figure provides quantitative evidence for the charging of the molecule as a function of bias and one can observe a total charge variation of about $0.05 e$ over the entire bias range of $4 \mathrm{~V}$. Interestingly, and despite the different terminating groups, the charge variation with bias is essentially identical for the two terminations.

\section{Influence of the tip-to-molecule electrostatic interaction over the transport}

As concluded in the previous section, the asymmetry of the conductance with respect to the bias direction found in our calculations is due to the asymmetry in the electronic coupling of the molecule to the leads. Interestingly a strong asymmetry was also observed in the experimental conduc-
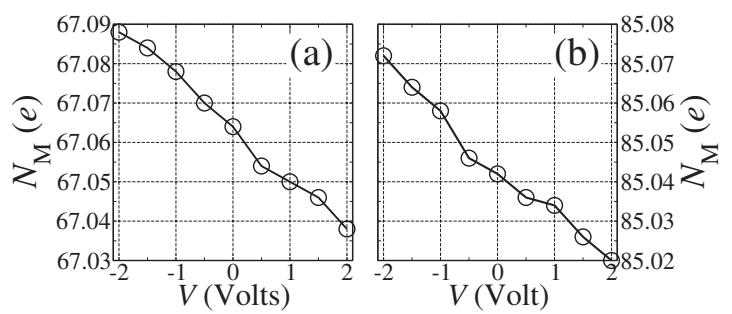

FIG. 16. Mulliken populations for decanethiol molecule terminate with (a) $\mathrm{CH}_{3}$ and (b) $\mathrm{CF}_{3}$ groups and attached to the gold surface. Note how in both cases the occupation of the molecule drops as the bias increases from negative to positive.

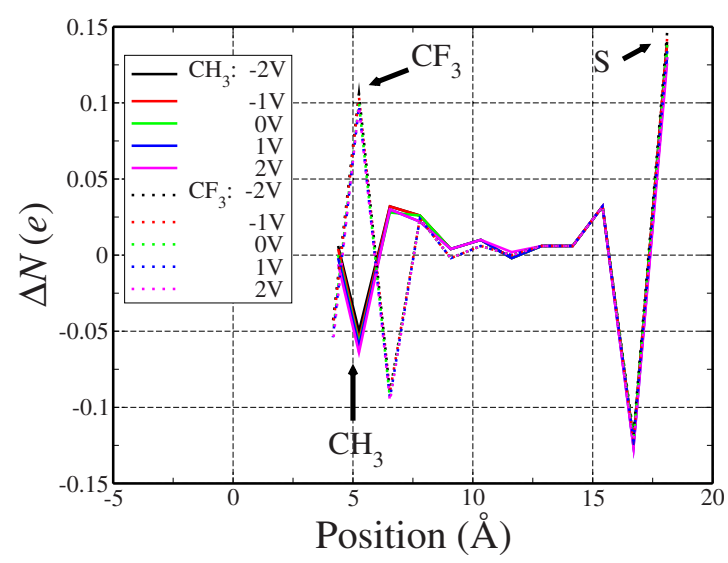

FIG. 17. (Color online) Net occupation of the atoms as the function of position along the axis of the molecule.

tance measurements for the same molecules. ${ }^{19}$ However, in the experiments, $\mathrm{CF}_{3}$-terminated molecules displayed a far more pronounced asymmetry than the $\mathrm{CH}_{3}$-terminated ones. This is in contrast with our findings where the asymmetry is similar, and in fact it is more pronounced for the $\mathrm{CH}_{3}$-terminated case. In agreement with the initial suggestion from Pflaum et al. ${ }^{19}$ here we argue that this additional asymmetry is not of electronic origin but rather is connected to the electrostatic interaction between the tip and the molecule under bias. This repositions the molecule with respect to the tip, effectively changing the tunneling current.

The main idea is illustrated schematically in Fig. 13(b). The large electronegativity of the $\mathrm{F}$ atoms in the $\mathrm{CF}_{3}$ group is expected to displace a substantial net charge toward the end of the molecule. This then interacts electrostatically with the surface charge of the STM tip, either repelling or attracting the molecule depending on the bias polarity. Such a charge displacement is illustrated in Fig. 17 where we show the charge excess with respect to neutrality, $\Delta N$, as calculated from the Mülliken population as a function of the position along the molecule axis. In our notation $\Delta N>0(\Delta N<0)$ indicates a negatively (positively) charged portion of the molecule. Substituting $\mathrm{H}$ by $\mathrm{F}$ atoms causes an increase in the occupation of the endmost carbon atom, although the $\mathrm{F}$ atoms show a net positive charge. Overall the total net charge on the $\mathrm{CF}_{3}$ group is negative (it posses extra electrons), whereas the total net charge on the $\mathrm{CH}_{3}$ group is positive. In addition the two end-groups produce an electrical dipole with opposite sign and different magnitude, with that associated with $\mathrm{CF}_{3}$ being the largest. Finally it is important to observe that by large the charge distribution over the molecule is not affected by the external electric field so that $\Delta N$ is almost bias independent for both the terminations.

We estimate the forces acting on the molecule by using simple classical electrostatic theory. In the case of $\mathrm{CH}_{3}$-terminated decanethiol we calculate a force of 3 $\times 10^{-3} \mathrm{eV} \AA^{-1}$ in the direction of the tip at the positive bias of $2 \mathrm{~V}$, and $2 \times 10^{-3} \mathrm{eV} \AA^{-1}$ in the direction of the substrate at the negative bias of $2 \mathrm{~V}$. These have to be put in relation with the elastic forces needed to stretch the molecule. DFT calculations performed using SIESTA demonstrate that the tipto-molecule electrostatic force at positive bias causes the 
molecule to stretch by about $0.002 \AA$, while at negative bias the compression is on the order of $0.001 \AA$ (at $\pm 2 \mathrm{~V})$. Similarly, the force acting on the $\mathrm{CF}_{3}$ group is calculated to be on the order of $3 \times 10^{-3} \mathrm{eV} \AA^{-1}$ in the direction of the substrate when a positive bias of $2 \mathrm{~V}$ is applied, and on the order of $4 \times 10^{-3} \mathrm{eV}^{-1}$ in the direction of the tip at a negative bias of $2 \mathrm{~V}$. These forces are in the opposite direction to those on the $\mathrm{CH}_{3}$ termination due to the opposite directions of the two dipoles. Again DFT calculations for $\mathrm{CF}_{3}$-decanethiol indicate that the force at positive bias causes the molecule to compress by about $0.001 \AA$, and the force at negative bias to stretch by $0.002 \AA$. These length changes are of the same magnitude for both types of end-group, but they occur at opposite bias directions due to the opposite direction of the electrostatic force in the two cases.

The stretching (or compressing) of the molecule under bias alters the tip-to-molecule separation, which in turn affects the magnitude of the current. Let us look at the $\mathrm{CH}_{3}$ case first. At positive bias the molecule is stretched so that its end is closer to the STM tip, the tip-to-molecule separation is reduced and the current will increase. In contrast at negative bias, the molecule is compressed and a reduction in the current is expected. Since the current obtained from our calculations at negative bias is larger than that for positive bias, such a change in molecule length would contribute to reducing the $I-V$ asymmetry in agreement with the experimental results. However, a quantitative estimate of the changes in the $I-V$ curve as a function of the molecule compression/ expansion reveals that molecular displacements of this tiny magnitude (a few percent of $\AA$ ) are insufficient to make a noticeable change to the $I-V$.

The results for $\mathrm{CF}_{3}$-terminated decanethiol are similar. At positive bias, the molecule is compressed so that the $\mathrm{CF}_{3}$ group is further away from the STM tip, while at negative bias the molecule is stretched and the tip-to-molecule separation is reduced. Since the current calculated at negative bias is larger than that at positive bias, the electrostatic interaction in this case has the effect of enhancing the asymmetry of the $I-V$ curves. This is also consistent with the experimental results, showing a larger asymmetry in the transport for the molecule terminated with the $\mathrm{CF}_{3}$ group. However, similar to the $\mathrm{CH}_{3}$-terminated case, the estimated molecule length change is insufficient to make a noticeable modification to the $I-V$ curve. Note that these negative estimates are all obtained by comparing the geometry at zero bias with that corresponding to the largest displacement (at $\pm 2 \mathrm{~V}$ ), so that they should be considered as the upper bound for the current changes. Therefore we can safely conclude that changes in molecule length can hardly be at the origin of the experimentally observed asymmetry in the $I-V$ curve.

A further possibility is to consider changes in the bonding angle between the molecule and the substrate. As mentioned before, decanethiols do not arrange vertically to the surface, but they rather form angles of 32 and $39^{\circ}$, respectively, for the $\mathrm{CH}_{3}$ and $\mathrm{CF}_{3}$ terminations. ${ }^{19}$ Hence it is likely that the interaction between the STM tip and the end-groups causes the molecule to rotate either away from or toward the STM tip, as shown in Fig. 18. The direction of the rotation depends again on the sign of the electrostatic force. However since the energy required to change the bonding angle is

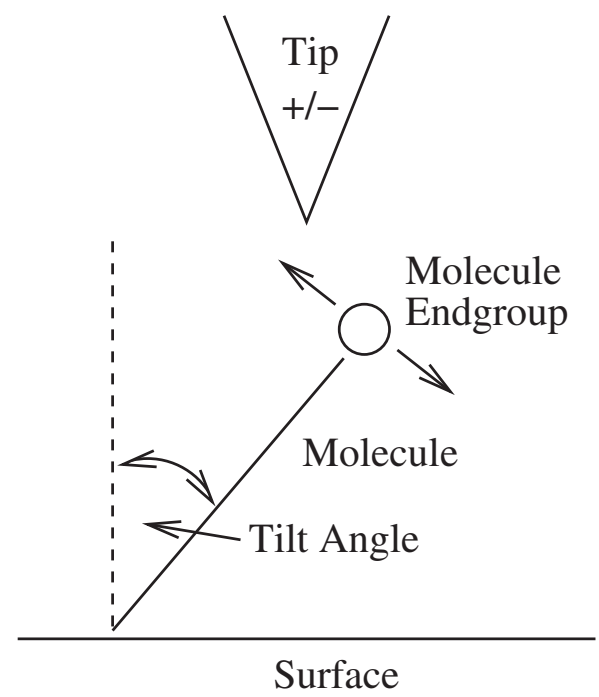

FIG. 18. Schematic illustration showing the tilting angle of the molecule over the Au surface and the rotation due to the interaction with the STM tip.

expected to be rather smaller than that required for an axial distortion, we expect changes in bond angle to produce larger changes in the tip-to-molecule separation.

Using the same electrostatic forces calculated before we estimate for the $\mathrm{CH}_{3}$ termination a $1^{\circ}$ rotation toward the STM tip at positive bias $(2 \mathrm{~V})$, and a $0.5^{\circ}$ rotation toward the surface at negative bias $(-2 \mathrm{~V})$. Similarly for the $\mathrm{CF}_{3}$ termination, the rotations are along the opposite direction with changes in the bond angle of $0.5^{\circ}$ toward the surface at positive bias and $1^{\circ}$ toward the STM tip at negative bias. These rotations are sufficient to alter the $I-V$ characteristics, as shown for both types of molecule in Fig. 19.

In the case of $\mathrm{CH}_{3}$ termination the calculated rotation is actually sufficient to suppress the asymmetry in the $I-V$ present because of the asymmetric coupling to the electrodes. The current at 1 Volt is now larger for positive bias than that at the same negative bias. In contrast for $\mathrm{CF}_{3}$-terminated

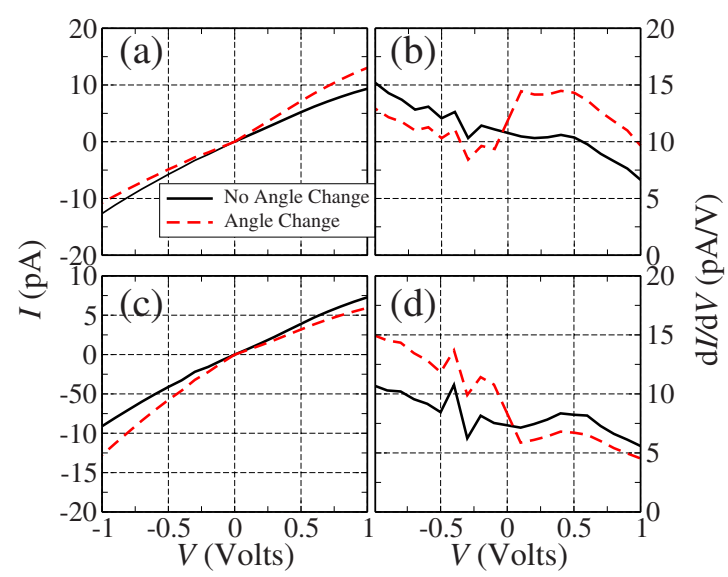

FIG. 19. (Color online) $I-V$ curves and differential conductance as a function of bias obtained both with and without considering the effects of the electrostatic-induced rotation of the molecule. Panels (a) and (b) show the $I-V$ and $d I / d V$ curves for the $\mathrm{CH}_{3}$ termination, and (c) and (d) show the same for the $\mathrm{CF}_{3}$ case. 
molecules, the rotation is such that the $I-V$ asymmetry already present is enhanced. Thus, when the electrostaticinduced rotation of the molecule is taken into account, the $I-V$ curve for the $\mathrm{CF}_{3}$-terminated molecule appears as more pronounced than that obtained for the $\mathrm{CH}_{3}$-terminated one, in semiquantitative agreement with experiments. ${ }^{19}$

In concluding we wish to point out a few possible sources of error in our calculations, which might affect a fully quantitative comparison with experiments. First we remark that the Au atoms of both the tip and the substrate are described at the $6 s$-only level. This provides a good description of the $\mathrm{Au}$ Fermi surface, thus of the transport properties, but usually it does not provide accurate interatomic forces. Second the calculated forces between the molecule and the tip certainly depend on the actual shape and detailed position of the tip. These are difficult to characterize with certainty and errors are therefore difficult to avoid. Finally the actual value of the tilting angle depends on the intermolecular interaction is the self-assembled monolayer, and thus the force required to change the angle would depend on the details of the molecular coverage (density, symmetry, bonding site, etc.).

\section{CONCLUSION}

We have demonstrated that the DFT-NEGF code SMEAGOL can be used to simulate STM-type experiments in the near to contact regime. This is under the working condition of the tip-to-sample distance being sufficiently small that the basis orbitals have not been artificially cut off (i.e., the vacuum region between the tip and the surface is still well described). SMEAGOL then allows us to investigate systems in which the tip interacts with the molecule, and to study effects of finite bias on the electronic structure of the molecule.
Calculations for alkanethiol molecules with STM-type arrangements show strong asymmetry in the $I-V$ curves, which can be explained by the asymmetry in the coupling to the two different electrodes (substrate and tip). However, the calculated asymmetry is similar for both $\mathrm{CH}_{3^{-}}$and $\mathrm{CF}_{3}$-terminated decanethiols, in contrast to the experimental measurements, showing a far stronger asymmetry for the case of $\mathrm{CF}_{3}$ termination. We have then investigated the original suggestion, which attributed the asymmetry to small configurational changes in the molecule under bias due to electrostatic interaction between the tip and the substrate. Our calculations demonstrate that changes to molecule length are too small and are unlikely to have any major impact on the $I-V$ curves; however, a rotation of the molecule may cause significant changes to the current.

As a final remark we point out that the results presented in this work have been obtained by using the LDA. However, LDA has several shortfalls which can strongly affect electron-transport calculations. In this case, approximate self-interaction corrections such as the atomic selfinteraction correction (ASIC) (Refs. 23, 32, and 33) are unlikely to offer a substantial improvement, since the conductance is due to off-resonance tunneling, and would not be particularly sensitive to the exact position of the molecular orbitals. However, for these molecules, an accurate calculation of the electric polarizability beyond the LDA might be important. ${ }^{34}$

\section{ACKNOWLEDGMENTS}

We thank G. Scoles for having driven our attention to the experiments discussed in this paper. This work was funded by Science Foundation of Ireland (Grant No. 07/IN.1/I945). Computational resources have been provided by the HEA IITAC project managed by the Trinity Center for High Performance Computing and by ICHEC.
*Present address: Dresden University of Technology, Dresden, Germany.

†sanvitos@tcd.ie

${ }^{1}$ G. Binnig, H. Rohrer, Ch. Gerber, and E. Weibel, Appl. Phys. Lett. 40, 178 (1982); Phys. Rev. Lett. 49, 57 (1982).

${ }^{2}$ F. Meier, L. Zhou, J. Wiebe, and R. Wiesendanger, Science 320 , 82 (2008).

${ }^{3}$ M. F. Crommie, C. P. Lutz, and D. M. Eigler, Science 262, 218 (1993).

${ }^{4}$ B. Xu and N. J. Tao, Science 301, 1221 (2003).

${ }^{5}$ X. Xiao, B. Xu, and N. J. Tao, Nano Lett. 4, 267 (2004).

${ }^{6}$ R. Temirov, A. Lassise, F. B. Anders, and F. S. Tautz, Nanotechnology 19, 065401 (2008).

${ }^{7}$ F. Pump, R. Temirov, O. Neucheva, S. Soubatch, S. Tautz, M. Rohlfing, and G. Cuniberti, Appl. Phys. A 93, 335 (2008).

${ }^{8}$ J. Bardeen, Phys. Rev. Lett. 6, 57 (1961).

${ }^{9}$ J. Tersoff and D. R. Hamann, Phys. Rev. Lett. 50, 1998 (1983); Phys. Rev. B 31, 805 (1985).

${ }^{10}$ A. R. Rocha, V. M. García-Suárez, S. Bailey, C. Lambert, J. Ferrer, and S. Sanvito, Nature Mater. 4, 335 (2005).
${ }^{11}$ A. R. Rocha, V. M. García-Suárez, S. Bailey, C. Lambert, J. Ferrer, and S. Sanvito, Phys. Rev. B 73, 085414 (2006); SMEAGOL is available at www.smeagol.tcd.ie.

${ }^{12}$ M. Brandbyge, J.-L. Mozos, P. Ordejón, J. Taylor, and K. Stokbro, Phys. Rev. B 65, 165401 (2002); J. Taylor, H. Guo, and J. Wang, ibid. 63, 245407 (2001).

${ }^{13}$ S. Datta, Electronic Transport in Mesoscopic Systems (Cambridge University Press, Cambridge, UK, 1995).

${ }^{14}$ H. Hohenberg and W. Kohn, Phys. Rev. 136, B864 (1964).

${ }^{15}$ W. Kohn and L. J. Sham, Phys. Rev. 140, A1133 (1965).

${ }^{16}$ J. M. Soler, E. Artacho, J. D. Gale, A. García, J. Junquera, P. Ordejón, and D. Sanchez-Portal, J. Phys.: Condens. Matter 14, 2745 (2002).

${ }^{17}$ J. Junquera, O. Paz, D. Sánchez-Portal, and E. Artacho, Phys. Rev. B 64, 235111 (2001).

${ }^{18}$ O. Paz and J. M. Soler, Phys. Status Solidi B 243, 1080 (2006).

${ }^{19}$ J. Pflaum, G. Bracco, F. Schreiber, R. Colorado, Jr., O. E. Shmakova, T. R. Lee, G. Scoles, and A. Kahn, Surf. Sci. 498, 89 (2002).

${ }^{20}$ Q. Sun and A. Selloni, J. Phys. Chem. A 110, 11396 (2006). 
${ }^{21}$ I. Rungger and S. Sanvito, Phys. Rev. B 78, 035407 (2008).

${ }^{22}$ A. R. Rocha and S. Sanvito, Phys. Rev. B 70, 094406 (2004).

${ }^{23}$ C. Toher and S. Sanvito, Phys. Rev. B 77, 155402 (2008).

${ }^{24}$ Note that this is the LDA HOMO-LUMO gap calculated from the Kohn-Sham eigenvalues. As such, in general it is not expected to be a good estimate of the actual experimental gap, which is the difference between the ionization potential and the electron affinity of the molecule. In this case however the junction is in a tunneling regime already at this LDA level and possible corrections are expected to produce only minor quantitative changes to the calculated transport properties.

${ }^{25}$ F. Picaud, A. Smogunov, A. Dal Corso, and E. Tosatti, J. Phys.: Condens. Matter 15, 3731 (2003).

${ }^{26}$ J. K. Tomfohr and O. F. Sankey, Phys. Rev. B 65, 245105 (2002).

${ }^{27}$ The sulphur-surface distance is defined as the distance between the $\mathrm{S}$ atom of the thiol group and the hollow position in the fcc (111) surface.

${ }^{28}$ H. Sellers, A. Ullman, Y. Shnidman, and J. E. Eilers, J. Am.
Chem. Soc. 115, 9389 (1993).

${ }^{29}$ J.-G. Wang, E. Prodan, R. Car, and A. Selloni, Phys. Rev. B 77, 245443 (2008).

${ }^{30}$ P. G. Piva, G. A. DiLabio, J. L. Pitters, J. Zikovsky, M. Rezeq, S. Dogel, W. A. Hofer, and R. A. Wolkow, Nature (London) 435, 658 (2005).

${ }^{31}$ Note that in our transport calculation the bias window is introduced by shifting the chemical potentials of both the tip and the substrate by $\pm e V / 2$. This is however completely equivalent to the standard STM setup where the sample potential is kept fixed and the voltage is applied to the tip. Note also that the definition of bias polarity in our work is opposite to that of Pflaum et al. (Ref. 19).

${ }^{32}$ C. Toher and S. Sanvito, Phys. Rev. Lett. 99, 056801 (2007).

${ }^{33}$ C. D. Pemmaraju, T. Archer, D. Sanchez-Portal, and S. Sanvito, Phys. Rev. B 75, 045101 (2007).

${ }^{34}$ C. D. Pemmaraju, S. Sanvito, and K. Burke, Phys. Rev. B 77, 121204(R), (2008). 